\title{
Comparison of Simulated and Measured Non-linear Ultrasound Fields
}

\author{
Du, Yigang; Jensen, Henrik; Jensen, Jørgen Arendt
}

\section{Published in:}

Proceedings of SPIE

Link to article, DOI:

$10.1117 / 12.877350$

Publication date:

2011

Document Version

Early version, also known as pre-print

Link back to DTU Orbit

Citation (APA):

Du, Y., Jensen, H., \& Jensen, J. A. (2011). Comparison of Simulated and Measured Non-linear Ultrasound Fields. In J. D'hooge, \& M. M. Doyley (Eds.), Proceedings of SPIE (Vol. 7968). SPIE - International Society for Optical Engineering. https://doi.org/10.1117/12.877350

\section{General rights}

Copyright and moral rights for the publications made accessible in the public portal are retained by the authors and/or other copyright owners and it is a condition of accessing publications that users recognise and abide by the legal requirements associated with these rights.

- Users may download and print one copy of any publication from the public portal for the purpose of private study or research.

- You may not further distribute the material or use it for any profit-making activity or commercial gain

- You may freely distribute the URL identifying the publication in the public portal 


\title{
Comparison of Simulated and Measured Non-linear Ultrasound Fields
}

\author{
Yigang Du ${ }^{1,2}$, Henrik Jensen ${ }^{2}$ and Jørgen Arendt Jensen ${ }^{1}$ \\ 1) Center for Fast Ultrasound Imaging, Department of Electrical Engineering, Bldg. 349, \\ Technical University of Denmark, DK-2800 Kgs. Lyngby, Denmark \\ 2) BK Medical Aps, Mileparken 34, DK-2730 Herlev, Denmark
}

\begin{abstract}
In this paper results from a non-linear AS (angular spectrum) based ultrasound simulation program are compared to water-tank measurements. A circular concave transducer with a diameter of 1 inch $(25.4 \mathrm{~mm})$ is used as the emitting source. The measured pulses are first compared with the linear simulation program Field II, which will be used to generate the source for the AS simulation. The generated non-linear ultrasound field is measured by a hydrophone in the focal plane. The second harmonic component from the measurement is compared with the AS simulation, which is used to calculate both fundamental and second harmonic fields. The focused piston transducer with a center frequency of $5 \mathrm{MHz}$ is excited by a waveform generator emitting a 6-cycle sine wave. The hydrophone is mounted in the focal plane $118 \mathrm{~mm}$ from the transducer. The point spread functions at the focal depth from Field II and measurements are illustrated. The FWHM (full width at half maximum) values are $1.96 \mathrm{~mm}$ for the measurement and $1.84 \mathrm{~mm}$ for the Field II simulation. The fundamental and second harmonic components of the experimental results are plotted compared with the AS simulations. The RMS (root mean square) errors of the AS simulations are $7.19 \%$ and $10.3 \%$ compared with the fundamental and second harmonic components of the measurements.
\end{abstract}

Keywords: Angular spectrum, Non-linear ultrasound simulation, Concave transducer, Water-tank measurement

\section{INTRODUCTION}

Simulations of ultrasound wave propagation can be used to optimize ultrasound imaging. For the research into non-linear ultrasound imaging, such a simulation program should be capable of calculating both linear and nonlinear ultrasound fields for arbitrary emitting apertures. Field $\mathrm{II}^{1,2}$ is a linear acoustic simulation program that can calculate both the emission and reception ultrasound fields for any kind of transducer, focusing, apodization, and attenuation linearly. The AS (angular spectrum) ${ }^{3,4}$ approach can be used to simulate both the linear and non-linear ultrasound fields. The feasibility of the combination of Field II and AS approach for the non-linear simulation and the comparison between Field II and the AS approach for a linear monochromatic system has been done by $\mathrm{Du}$ and Jensen ${ }^{5}$ in 2008 . Furthermore, the simulation of pulsed linear ultrasound fields using the AS approach was achieved ${ }^{6}$ in 2009. The simulation of non-linear ultrasound fields was studied by Taylor and Jensen ${ }^{7,8}$ using an OS (operator splitting) method ${ }^{9,10}$ based on Field II. A non-linear simulation software Abersim ${ }^{11,12}$ based on both the AS approach and OS method was released in 2008. However, if the simulated point is far from the acoustic source, the calculation speed becomes extremely slow when using the OS method, because the computation is based on a source, which should be very close to the simulated point according to the Poisson solution. ${ }^{13}$ Thus, the calculation will be executed hundreds of times for a long distance propagation.

In this paper, the AS approach based on Field II is used to calculate the second harmonic ultrasound field. Only one step is needed no matter how far the simulated point is from its source. The full equations for the fundamental and second harmonic components using the AS approach are derived in Section 2 (Theory). The

Further author information: (Send correspondence to Yigang Du)

Yigang Du: E-mail: yd@elektro.dtu.dk, Telephone: +45 45253915

Henrik Jensen: E-mail: hj@bkmed.dk, Telephone: +45 44528156

Jørgen Arendt Jensen: E-mail: jaj@elektro.dtu.dk, Telephone: +45 45253924 
implementation of the equations and set-up of measurements are shown in Section 3 (Method). Finally, the results from the AS and Field II simulations for both the fundamental and second harmonic components of the ultrasound fields are compared with the water-tank hydrophone measurements. The comparisons of the results between the theory and experiments are illustrated quantitatively and graphically in Section 4 (Results). The discussion of future work and conclusion are made in the last two sections.

\section{THEORY}

The non-linear acoustic wave propagation can be described by the Westervelt equation ${ }^{14}$

$$
\left(\nabla^{2}-\frac{1}{c_{0}^{2}} \frac{\partial^{2}}{\partial t^{2}}\right) p+\frac{\delta}{c_{0}^{4}} \frac{\partial^{3} p}{\partial t^{3}}+\frac{\beta}{\rho_{0} c_{0}^{4}} \frac{\partial^{2} p^{2}}{\partial t^{2}}=0,
$$

where $c_{0}$ and $\rho_{0}$ are speed of sound and density of medium, and assuming that both of them are constant for a given medium. $\nabla^{2}$ represents $\partial^{2} / \partial x^{2}+\partial^{2} / \partial y^{2}+\partial^{2} / \partial z^{2} \cdot p$ is the acoustic pressure and a function of time $t$ and space $x, y$ and $z . \beta$ in the non-linear term describes the extent of non-linear effect in the medium. $\delta$ in the attenuation term is the diffusivity of the attenuating medium. The Westervelt equation is derived from the continuity equation, Euler force equation, and the state equation of second order's Taylor expansion. It is assumed that the small amplitude fluctuation makes it possible to ignore the third and higher order terms for the Taylor expansion. The second assumption is that the sound propagates in water, so that the attenuation term can be neglected, since the attenuation of water is very low. The total sound pressure is the sum of the fundamental and second harmonic components, since the third and higher order components are not taken into consideration,

$$
p=p_{1}+p_{2}
$$

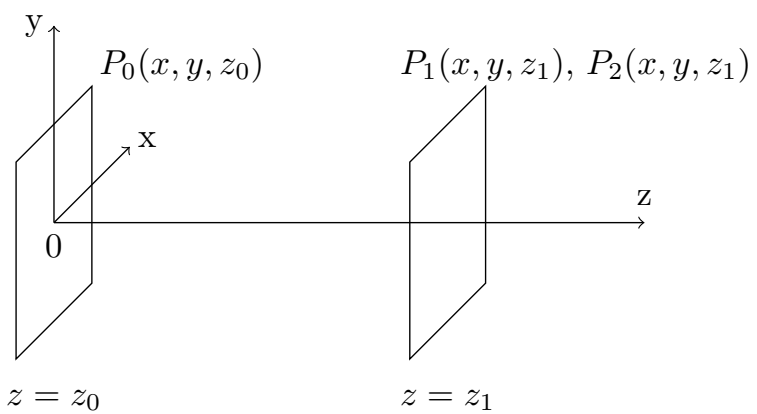

Figure 1. Schematic view of the angular spectrum approach. The acoustic plane at $z_{0}$ is the source plane, and the acoustic plane at $z_{1}$ is the calculated plane. The acoustic pressure for the fundamental component $P_{1}$ and second harmonic component $P_{2}$ are calculated based on the source $P_{0}$ and the distance between two planes. The distance between the source plane and calculated plane is $z_{1}-z_{0}$.

Using the separate variable solution, ${ }^{15-17}$ the acoustic pressure of the $n$th harmonic is

$$
p_{n}(x, y, z, t)=\frac{1}{2} P_{n}(x, y, z) e^{-j n \omega t}+c . c ., \quad n=1,2
$$

where $P_{n}$ is the amplitude of the sound wave of the $n$th harmonic, $\omega$ is the fundamental angular frequency given by the source plane and c.c. means the complex conjugate of the preceding term $\left(\right.$ c.c. $\left.=\left(\frac{1}{2} P_{n}(x, y, z) e^{-j n \omega t}\right)^{*}\right)$. Substituting (2) and (3) into (1) without the loss term leads to

$$
\left(\nabla^{2}+k^{2}\right) P_{1}+\left(\nabla^{2}+4 k^{2}\right) P_{2} e^{-j \omega t}=\frac{2 \beta k^{2}}{\rho_{0} c_{0}^{2}} P_{1}^{2} e^{-j \omega t},
$$

where $k$ is wave number and given by $k=\omega / c_{0}$, and $P_{1}^{2}$ is obtained by the third assumption $p_{1} \gg p_{2} \Longrightarrow$ $\left(p_{1}+p_{2}\right)^{2} \approx p_{1}^{2}$. Thus, (4) can be divided into two equations, since the first term of the left hand side of (4) is 
not a function of time $t$.

$$
\begin{aligned}
\left(\nabla^{2}+k^{2}\right) P_{1} & =0, \\
\left(\nabla^{2}+4 k^{2}\right) P_{2} & =\frac{2 \beta k^{2}}{\rho_{0} c_{0}^{2}} P_{1}^{2} .
\end{aligned}
$$

A linear AS approach ${ }^{3,18}$ being applied to (5) gives

$$
\hat{P}_{1}\left(k_{x}, k_{y}, z_{1}\right)= \begin{cases}\hat{P}_{0}\left(k_{x}, k_{y}, z_{0}\right) e^{-j\left(z_{1}-z_{0}\right) \sqrt{k^{2}-k_{x}^{2}-k_{y}^{2}}}, & k^{2}>k_{x}^{2}+k_{y}^{2} \\ \hat{P}_{0}\left(k_{x}, k_{y}, z_{0}\right) e^{-\left(z_{1}-z_{0}\right) \sqrt{k_{x}^{2}+k_{y}^{2}-k^{2}}}, & k^{2} \leq k_{x}^{2}+k_{y}^{2},\end{cases}
$$

where $\hat{P}_{0}$ and $\hat{P}_{1}$ are the $2 \mathrm{D}(x-y)$ spatial Fourier transform of $P_{0}$ and $P_{1} . k_{x}$ and $k_{y}$ are the transverse wave numbers along $x$ and $y$ directions as shown in Fig. 1 . The solution to (6) is derived by taking the $2 \mathrm{D}$ spatial inverse Fourier transform for $\hat{P}_{1}$ and using the convolution theorem ${ }^{19,20}$

$$
f * g=\mathbf{F}\left\{\mathbf{F}^{-1}\{f\} \cdot \mathbf{F}^{-1}\{g\}\right\},
$$

where $\mathbf{F}\{\}$ and $\mathbf{F}^{-1}\{\}$ denote the 2D $(x-y)$ spatial Fourier and inverse Fourier transforms, respectively. Then (6) can be expressed as

$$
\left(\nabla^{2}+4 k^{2}\right) P_{2}=\mathbf{F}^{-1}\left\{\frac{\beta k^{2}}{2 \pi^{2} \rho_{0} c_{0}^{2}} \iint \hat{P}_{1}\left(k_{x}^{\prime}, k_{y}^{\prime}, z_{1}\right) \hat{P}_{1}\left(k_{x}-k_{x}^{\prime}, k_{y}-k_{y}^{\prime}, z_{1}\right) d k_{x}^{\prime} d k_{y}^{\prime}\right\} .
$$

An ordinary differential equation with an inhomogeneous integral term is obtained after the $2 \mathrm{D}(x-y)$ spatial Fourier transform on both sides of (9), with the help of (7)

$$
\left(\frac{d^{2}}{d z^{2}}+k_{z 2}^{2}\right) \hat{P}_{2}\left(k_{x}, k_{y}, z_{1}\right)=\frac{\beta k^{2}}{2 \pi^{2} \rho_{0} c_{0}^{2}} M\left(k_{x}, k_{y}, z_{1}\right),
$$

where

$$
\begin{aligned}
M\left(k_{x}, k_{y}, z_{1}\right) & =\iint e^{-j\left(z_{1}-z_{0}\right)\left(k_{z}^{\prime}+k_{z}^{\prime \prime}\right)} \hat{P}_{0}\left(k_{x}^{\prime}, k_{y}^{\prime}, z_{0}\right) \hat{P}_{0}\left(k_{x}-k_{x}^{\prime}, k_{y}-k_{y}^{\prime}, z_{0}\right) d k_{x}^{\prime} d k_{y}^{\prime}, \\
k_{z 2} & =\sqrt{4 k^{2}-k_{x}^{2}-k_{y}^{2}}, \\
k_{z}^{\prime} & =\sqrt{k^{2}-\left(k_{x}^{\prime}\right)^{2}-\left(k_{y}^{\prime}\right)^{2}} \\
k_{z}^{\prime \prime} & =\sqrt{k^{2}-\left(k_{x}-k_{x}^{\prime}\right)^{2}-\left(k_{y}-k_{y}^{\prime}\right)^{2}} .
\end{aligned}
$$

Finally, the solution to (10) gives ${ }^{16,21}$

$$
\hat{P}_{2}\left(k_{x}, k_{y}, z_{1}\right)=\frac{\beta k^{2}}{2 \pi^{2} \rho_{0} c_{0}^{2}} \iint \hat{P}_{0}\left(k_{x}^{\prime}, k_{y}^{\prime}, z_{0}\right) \hat{P}_{0}\left(k_{x}-k_{x}^{\prime}, k_{y}-k_{y}^{\prime}, z_{0}\right) \frac{\left(e^{-j\left(z_{1}-z_{0}\right)\left(k_{z}^{\prime}+k_{z}^{\prime \prime}\right)}-e^{-j\left(z_{1}-z_{0}\right) k_{z 2}}\right)}{k_{z 2}^{2}-\left(k_{z}^{\prime}+k_{z}^{\prime \prime}\right)^{2}} d k_{x}^{\prime} d k_{y}^{\prime} .
$$

This is the so-called non-linear AS solution to the Westervelt equation and the wave number $k$ in (15) is a constant for monochromatic fields. The relation between the second harmonic pressure at the plane $z=z_{1}$ and the fundamental pressure at the plane $z=z_{0}$ is given by this equation.

\section{METHOD}

To make a comparison between the AS approach and water-tank measurements, the same transducer is used as the emitting source in simulations and measurements, respectively. The specification of the transducer is shown in Table 1. In the water tank, a focused single element Panametrics ultrasonic transducer V307 and a PVDF (polyvinylidene fluoride) ultrasonic hydrophone probe MH28-5 made by Force Technology Aps are used for the transmission and detection. The acoustic plane at the focal depth $118 \mathrm{~mm}$ from the transducer surface is simulated and measured. 
Table 1. Parameters of the transducer

\begin{tabular}{l||c|c}
\hline Transducer parameters & Simulations & Measurements \\
\hline Center frequency $f_{0}$ & $5 \mathrm{MHz}$ & $5 \mathrm{MHz}$ \\
Sampling frequency $f_{s}$ & $100 \mathrm{MHz}$ & $100 \mathrm{MHz}$ \\
Excitations & 6-cycle sine wave & 6-cycle sine wave \\
Geometrical focal point & $118 \mathrm{~mm}$ & $118 \mathrm{~mm}$ \\
Diameter & 1 inch $(25.4 \mathrm{~mm})$ & 1 inch $(25.4 \mathrm{~mm})$ \\
Bandwidth & - & $1.5-9.5 \mathrm{MHz}$ \\
Sound speed in water & $1480 \mathrm{~m} / \mathrm{s}$ & -
\end{tabular}

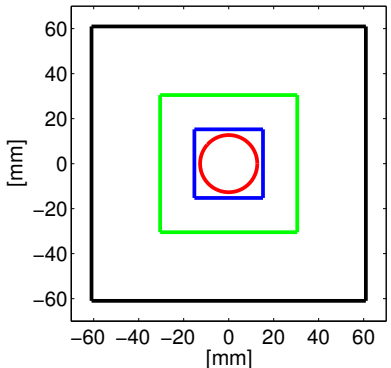

(a) Size: transducer vs virtual sources

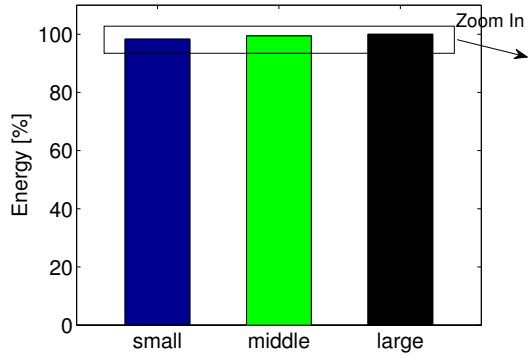

(b) Energy of different virtual sources

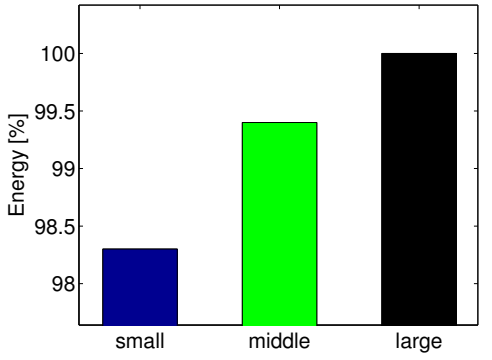

(c) Zoom-In of the middle figure

Figure 2. Left figure: Three size virtual sources with different colors are shown in square. The size of the transducer is shown in red circle. The virtual source $P_{0}\left(x, y, z_{0}\right)$ is $5 \mathrm{~mm}$ from the transducer surface. Middle figure: The energy for different sizes of the virtual source are shown. Right figure: Zoom-In on the top of the middle figure shows the differences of the energy among different size sources.

\subsection{Simulation}

The AS solution to the Westervelt equation in (15) is implemented using Matlab 7.8.0 (R2009a). The transducer as shown in Table 1 is defined by Field II. $P_{0}\left(x, y, z_{0}\right)$ is calculated by the Field II function "calc_hp", where $z_{0}=5$ $\mathrm{mm}$ from the transducer surface as shown in Fig. 1 . $P_{0}\left(x, y, z_{0}\right)$ is discretized by $412 \times 412$ points. The interval between each nearby two points is set to $\lambda / 4$ according to the Nyquist-Shannon sampling theorem ${ }^{22}$ to avoid aliasing, where $\lambda$ is the wavelength and given by $\lambda=c_{0} / f_{0} . P_{0}\left(x, y, z_{0}\right)$ covers a square area of $30.5 \times 30.5 \mathrm{~mm}^{2}$ in contrast to the circular surface of the transducer with a diameter of $25.4 \mathrm{~mm}$. The used size of $P_{0}\left(x, y, z_{0}\right)$ in the implementation is the smallest blue square as shown in Fig. 2a. The assumption is that, after $5 \mathrm{~mm}$ propagation from the transducer, the plane $P_{0}\left(x, y, z_{0}\right)$ is used as the virtual source to calculate the second harmonic pressure for other planes. This virtual source should be large enough to contain almost all the energy of the acoustic wave coming from the transducer. It is a compromise that the larger the virtual source plane is, the more energy it contains, the more accurate results should be obtained but the more computation time is required.

Fig. $2 \mathrm{~b}$ shows the energy contained by the different size planes. The energy is calculated by the Field II program. If assuming that the black square is large enough and containing all the acoustic energy, then the blue small and green middle squares contain $98.3 \%$ and $99.4 \%$ energy of it, respectively as shown in Fig. 2c. Nearly all the energy is contained within the blue small square.

Although it can be more accurate by using the green middle square, twice the size of the blue small one will make the computation 4 times as heavy. Therefore, to save time, the blue small square is used as the virtual source and implemented in the paper. $\hat{P}_{0}\left(k_{x}, k_{y}, z_{0}\right)$ in $(15)$ can be obtained by taking the $2 \mathrm{D}(x-y)$ spatial Fourier transform for the virtual source $P_{0}\left(x, y, z_{0}\right)$, which has to be zero-padded ${ }^{23}$ to avoid the circular convolution before the Fourier transform. The second harmonic pressure $\hat{P}_{2}\left(k_{x}, k_{y}, z_{1}\right)$ at the focal plane is obtained by implementing (15). $z_{1}-z_{0}$ is $113 \mathrm{~mm}$ in (15) since $z_{1}=118 \mathrm{~mm}$ corresponding to the focal plane. It is assumed that the emitted acoustic source is monochromatic. Therefore, the wave number $k$ in (15) is a constant and equals $2 \pi f_{0} / c_{0}$, where $f_{0}=5 \mathrm{MHz}$ and $c_{0}=1480 \mathrm{~m} / \mathrm{s}$ in the simulation. 


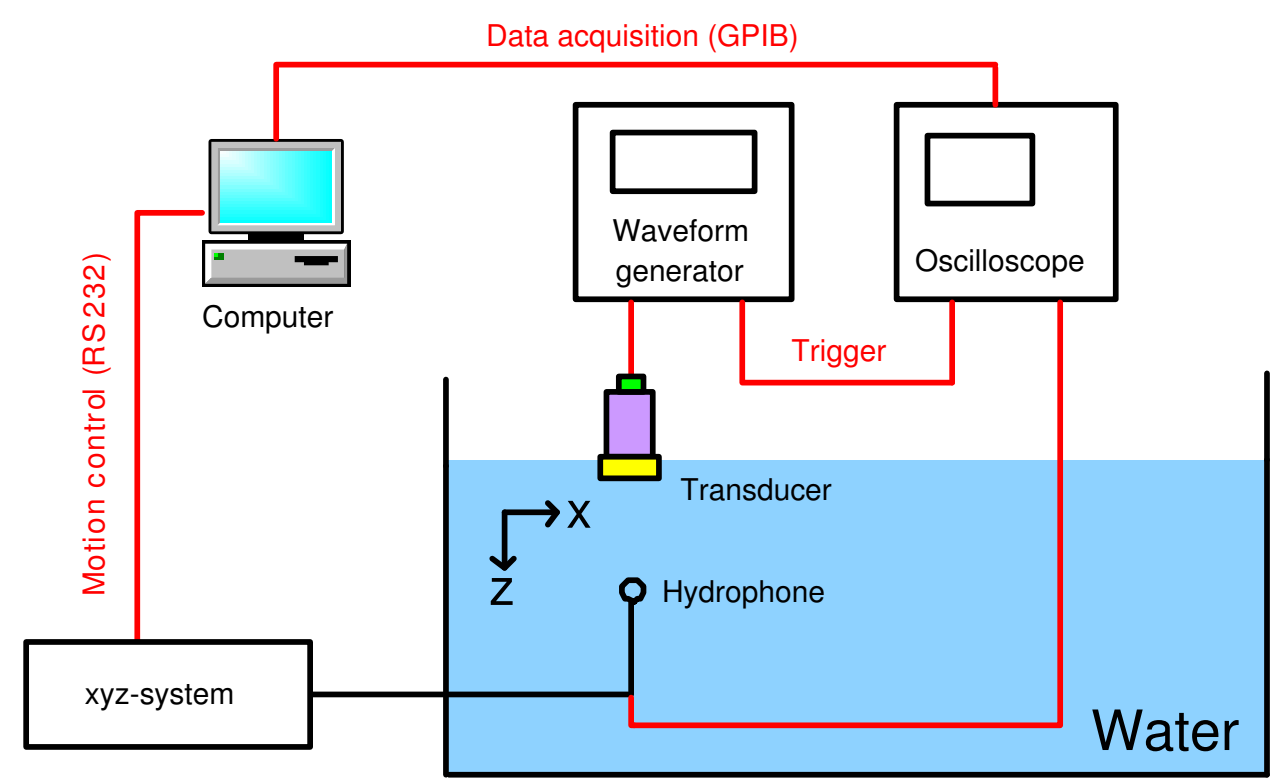

Figure 3. Systematic view of the water-tank hydrophone measurement.
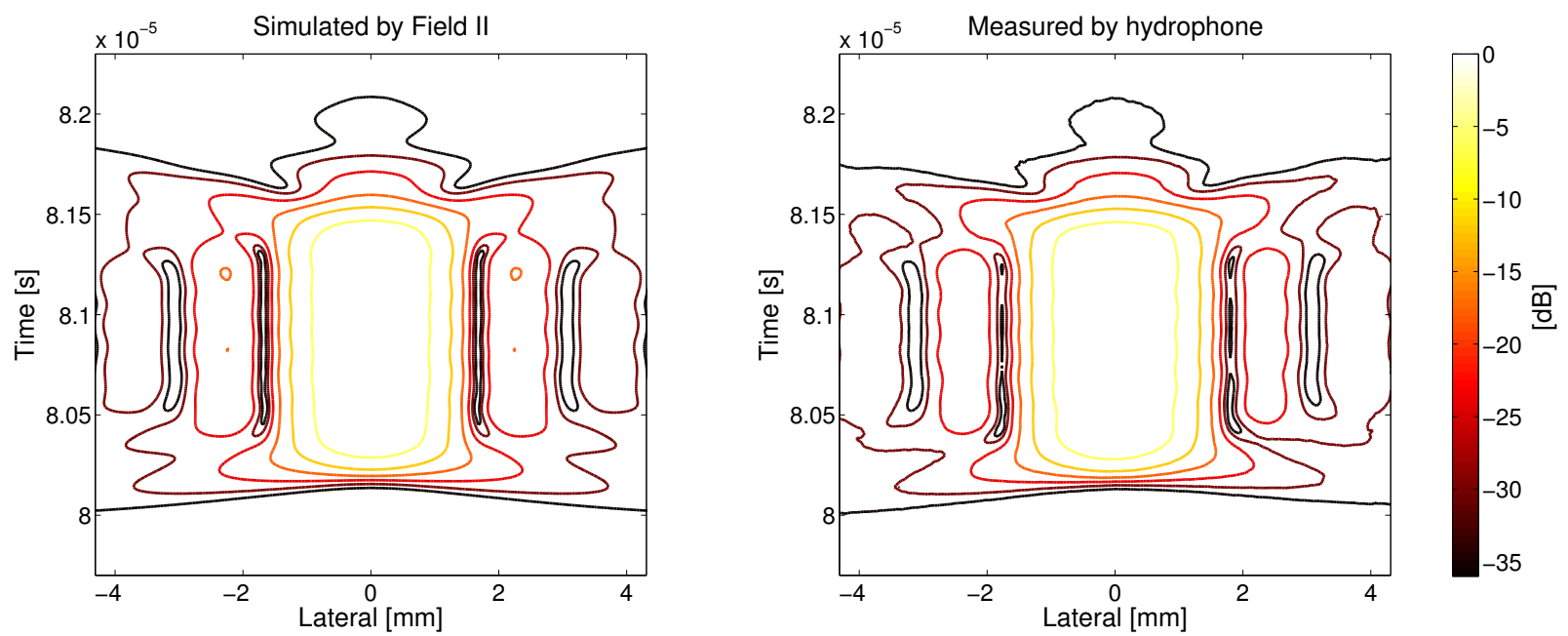

Figure 4. Point spread function at the focal plane, $118 \mathrm{~mm}$ from the transducer surface. The difference between nearby two colored lines is $6 \mathrm{~dB}$.

\subsection{Measurement}

The measurement is set up as shown in Fig. 3. The hydrophone is mounted in the focal plane, which is $118 \mathrm{~mm}$ away from the transducer surface. It moves in the $x$-axis (lateral direction) and is controlled by the xyz-system. The transducer is fixed and excited by the waveform generator, which has a trigger pulse to an oscilloscope and synchronized with the excitation of the transducer. This makes the transducer emission and the oscilloscope data recording start at the same time. For each point in a fixed position, one acoustic pulse with a time line can be obtained. By moving the hydrophone along the lateral direction and measuring the pulses at different positions, an emitted field for the focal plane is generated and stored in the oscilloscope. All these measurement activities can be controlled by a computer with Matlab scripts through GPIB (general purpose interface bus) and RS232 interface connections. 


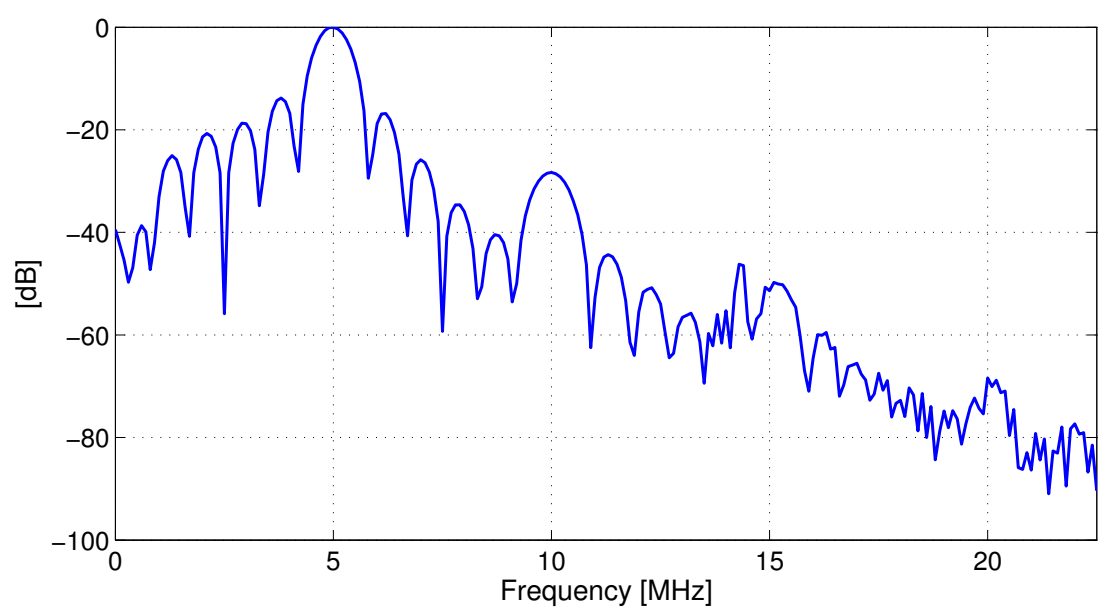

Figure 5. Mean spectrum of 240 measured pulses at different positions of the focal plane. Fundamental: $5 \mathrm{MHz}$, Second harmonic: $10 \mathrm{MHz}$, Third harmonic: $15 \mathrm{MHz}$, Fourth harmonic: $20 \mathrm{MHz}$. The bandwidth for the hydrophone is around $1 \mathrm{MHz}$ to $20 \mathrm{MHz}$. Therefore, the measured frequency components higher than $20 \mathrm{MHz}$ are not taken into account.

Table 2. Pressure in $\mathrm{dB}$ for the first four harmonic components

\begin{tabular}{c|l|c|c}
\hline & & Frequency $[\mathrm{MHz}]$ & Pressure $[\mathrm{dB}]$ \\
\hline 1 & Fundamental component & $5 \mathrm{MHz}$ & 0 \\
2 & Second harmonic component & $10 \mathrm{MHz}$ & $-28.3 \mathrm{~dB}$ \\
3 & Third harmonic component & $15 \mathrm{MHz}$ & $-51.4 \mathrm{~dB}$ \\
4 & Fourth harmonic component & $20 \mathrm{MHz}$ & $-68.4 \mathrm{~dB}$ \\
\hline
\end{tabular}

\section{RESULTS}

The point spread function of the measured pulses at the focal plane is compared to the simulated results from Field II as shown in Fig. 4. The measurement is located $118 \mathrm{~mm}$ from the transducer surface. The nonlinear effect is generated through the propagation in water. Therefore, the measured pulses actually include the second, third and even more higher harmonic components. However, the results from Field II are pure linear simulations. The comparison from Fig. 4 shows visually comparable results between the linear simulation of Field II and measurements even with non-linear effects. The FWHM (full width at half maximum) values are $1.96 \mathrm{~mm}$ for the measurement and $1.84 \mathrm{~mm}$ for the Field II simulation. The error in percentage between them is $6.24 \%$. Fig. 5 shows the mean spectrum of 240 measured pulses along the center line at the focal plane. The values of the pressure amplitude for the first four harmonic components are shown in Table 2. The higher harmonic component whose frequency is more than $20 \mathrm{MHz}$, will not be taken into consideration due to the bandwidth (1 MHz to $20 \mathrm{MHz}$ approximately) of the hydrophone used in the measurement. This shows that the fundamental component in the measured pulse is much larger than the second harmonic, and the higher harmonics are negligible compared to the fundamental and second harmonic components. From this study, the assumptions, $p=p_{1}+p_{2}$ and $p_{1} \gg p_{2}$ in the theory of AS approach are satisfied, and the calculated $p_{2}$ in the AS simulation becomes possible to compare with the second harmonic component from the measured pulse.

Table 3. RMS errors between AS approach, Field II and measurements

\begin{tabular}{r|l|l|c||c}
\hline & $P_{\mathrm{c}}\left(i_{x}, f\right)$ & $P_{\text {ref }}\left(i_{x}, f\right)$ & Frequency & RMS-error $(f)$ \\
\hline 1 & Field II & Measurement & Fundamental 5 MHz & $7.39 \%$ \\
2 & AS approach & Field II & Fundamental 5 MHz & $0.67 \%$ \\
3 & AS approach & Measurement & Fundamental 5 MHz & $7.19 \%$ \\
4 & AS approach & Measurement & Second harmonic $10 \mathrm{MHz}$ & $10.3 \%$ \\
\hline
\end{tabular}




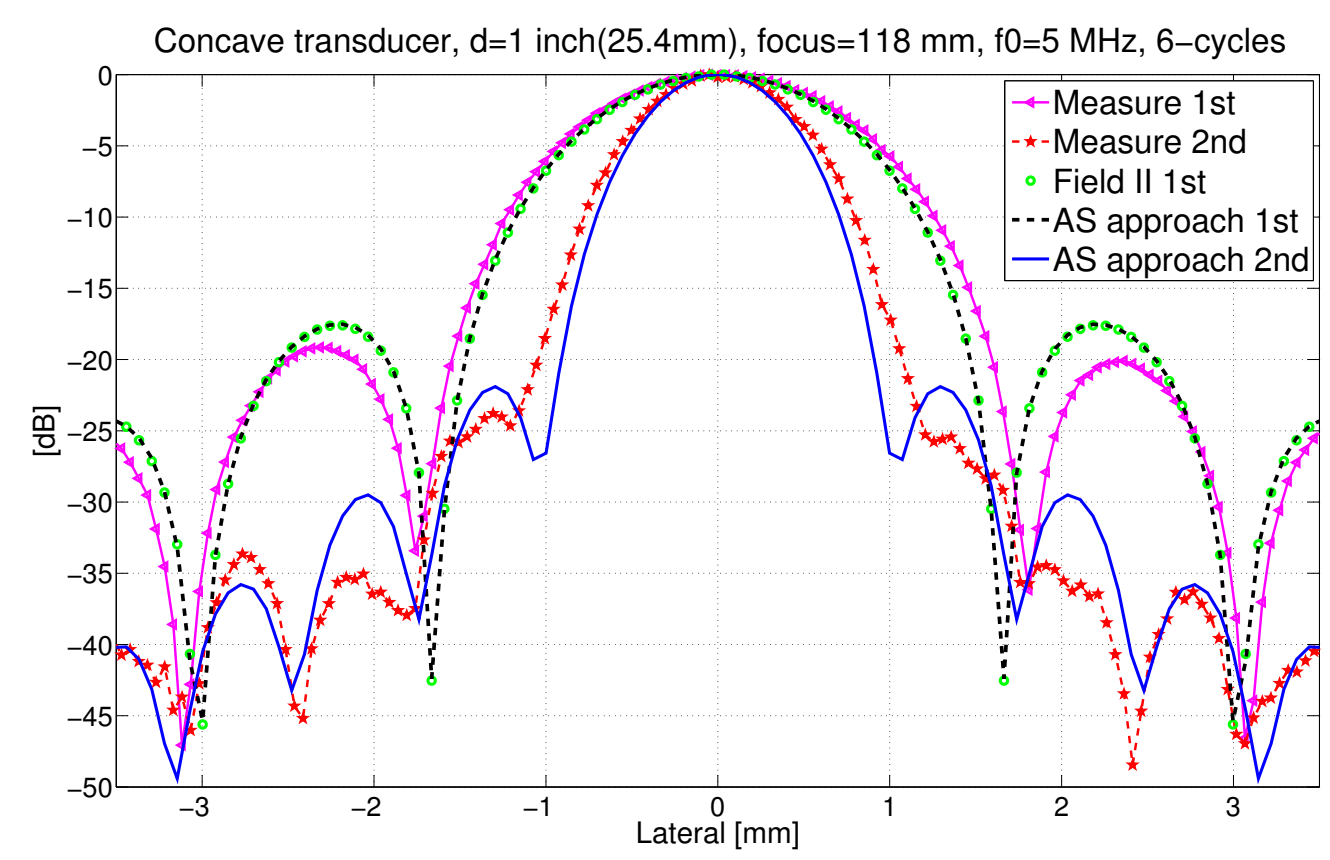

Figure 6. Comparisons between measurements and simulations of the fundamental and second harmonic components using the AS approach and Field II. These are calculated and measured at the focal distance $(z=118 \mathrm{~mm}$ from the transducer surface). The results obtained from each method or measurement for the fundamental or second harmonic components are normalized to themselves, which means the absolute difference between the fundamental and second harmonic components is not shown in the figure. In the legend, 1st means fundamental frequency component at $5 \mathrm{MHz}$ and 2 nd means second harmonic component at $10 \mathrm{MHz}$.

To avoid the overlapping between fundamental and second harmonic components, a 6-cycle $5 \mathrm{MHz}$ sinusoid is used as the excitation of the transducer to get a narrow bandwidth for the signals. In the measurement, the frequency components at $5 \mathrm{MHz}$ and $10 \mathrm{MHz}$ are filtered out from the spectrum of the measured pulses, respectively. The simulated fundamental frequency component at $5 \mathrm{MHz}$ using the AS approach based on a source plane generated by Field II is obtained. The second harmonic component at $10 \mathrm{MHz}$ is further calculated by the AS solution to the Westervelt equation. The comparisons of the ultrasound pressure at the fundamental frequency $5 \mathrm{MHz}$ and second harmonic frequency $10 \mathrm{MHz}$ between water-tank measurements and simulations using the AS approach and Field II are shown in Fig. 6. The quantitative comparisons are made by the RMS (root mean square) errors

$$
\operatorname{RMS}-\operatorname{error}(f)=\sqrt{\frac{\sum_{i_{x}=0}^{N=93}\left[P_{\mathrm{c}}\left(i_{x}, f\right)-P_{\text {ref }}\left(i_{x}, f\right)\right]^{2}}{\sum_{i_{x}=0}^{N=93} P_{\text {ref }}\left(i_{x}, f\right)^{2}}} \times 100 \%,
$$

where $P_{\mathrm{c}}\left(i_{x}, f\right)$ is the discrete form of the pressure simulated by the AS approach or Field II program at the focal plane $\left(y=0, z_{1}=118 \mathrm{~mm}\right)$ and a function distributed along $x$-axis (lateral direction) where $i_{x}$ is from 0 to 93 corresponding to $(-3.5 \sim 3.5) \mathrm{mm}$, and $f$ can be $5 \mathrm{MHz}$ or $10 \mathrm{MHz}$ in this case. $P_{\text {ref }}\left(i_{x}, f\right)$ is the reference pressure measured in the water tank or simulated by the Field II program at the same location for the same frequency components. The RMS errors are shown in Table 3. Comparison 2 reveals that the two simulation methods give very similar results. By use of the AS approach based on the Field II program, the generation of second harmonic component during the propagation of the acoustic pressure can be studied in the simulation. 


\section{DISCUSSION OF FUTURE WORK}

In medical ultrasound imaging system, a short pulse with high bandwidth of the spectrum is generally used as the emitted source in the transducer. To obtain a second harmonic point spread function similar to the linear simulation shown in Fig. 4 by Field II, the monochromatic simulation is inadequate. Therefore, (15) with non constant $k$ has to be implemented for the future work in order to simulate the second harmonic pulsed fields.

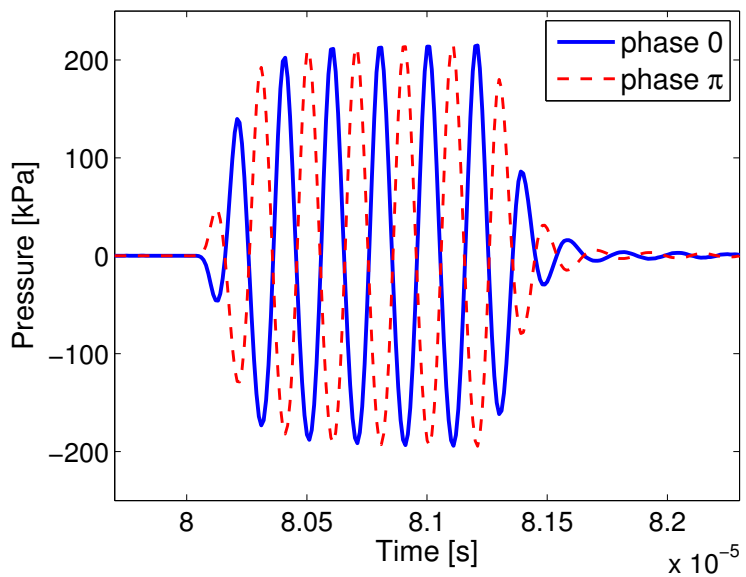

(a) Pulses obtained from inverted excitations

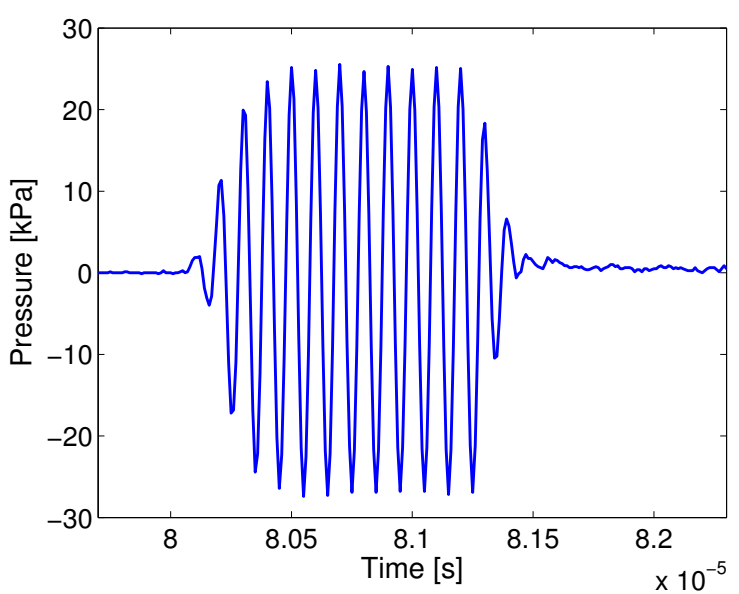

(b) Pulse after pulse inversion, $f_{0}=5 \mathrm{MHz}$

Figure 7. Two pulses in the left figure are measured at the focal point which is $118 \mathrm{~mm}$ from the transducer surface by sending two inverted pulses for the excitation, respectively. The pulse in the right figure is the sum of those two pulses from the left. The maximum value of the amplitude, for the measured pulse is around $214.5 \mathrm{kPa}$, for the second harmonic pulse after the filtration by the pulse inversion technique is $13.7 \mathrm{kPa}$ approximately. Note that the amplitude for the second harmonic pulse shown in the right figure is doubled.

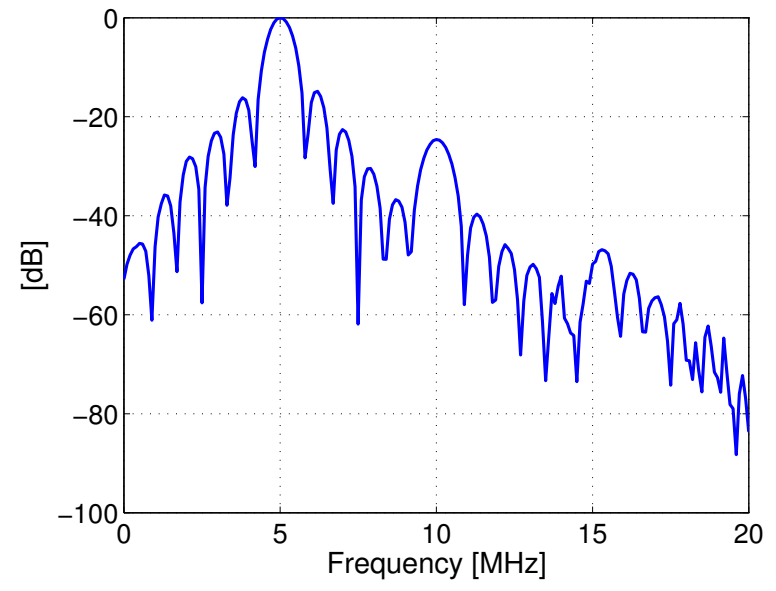

(a) Spectrum of the normal pulse

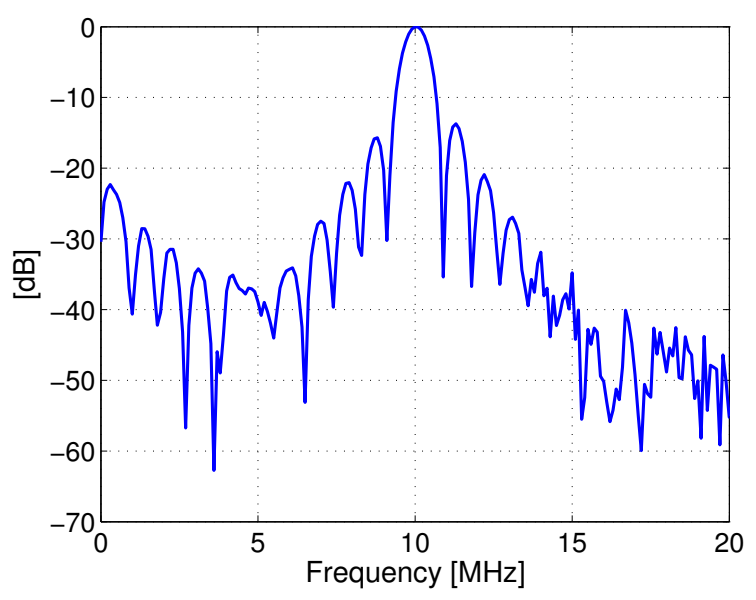

(b) Spectrum after using pulse inversion

Figure 8. Comparison of the spectrums before and after using pulse inversion technique is shown. The second harmonic component is down to $-24.6 \mathrm{~dB}$ compared to the fundamental as shown in the left figure.

The measured second harmonic pulses can be obtained by use of the pulse inversion technique. ${ }^{24-26}$ The signal at the focal point in the coordinate $\left(x=0, y=0, z_{1}=118\right) \mathrm{mm}$ as shown in Fig. 1 is measured twice. First a normal pulse is used as the excitation for the transducer, and secondly an inverted pulse with the phase of 180 degree is applied. The measured two pulses at the focal point are shown in Fig. 7a. The sum of these two pulses 
are shown in Fig. 7b. The fundamental components are canceled out and the second harmonic components are doubled. Fig. 8 shows the spectrums of the pulses with and without pulse inversion. A significant fundamental suppression after using pulse inversion can be found in Fig. 8b. In this way, the second harmonic pulse can be extracted from the measurement even for the wide bandwidth signals with the overlapped spectrum of the fundamental and second harmonic components.

\section{CONCLUSION}

The measured point spread function is compared with the linear simulation of Field II program. The comparison reveals that the non-linear effect in the measurement is low enough, so that the assumption $p_{1} \gg p_{2}$ is satisfied and can be used in the AS approach in principle. Then the Field II program is used to initialize the same transducer as used in the measurement to generate the source plane for the AS approach. The fundamental component is calculated by the AS approach, Field II, and measured under water by a hydrophone, respectively. The RMS error between the two simulations is only $0.67 \%$ and compared with measurements, the two simulations show similar RMS errors, which are $7.39 \%$ for Field II and $7.19 \%$ for the AS approach. This ensures that the second harmonic component can be simulated by the AS approach based on the source calculated by the Field II program. The AS solution to the Westervelt equation for calculating the second harmonic component of ultrasound fields is implemented for a monochromatic field. The RMS error of the second harmonic component for the AS simulation is $10.3 \%$ compared with the measurement.

\section{ACKNOWLEDGMENTS}

This work is sponsored by grant 9700883, 9700563 and 26-04-0024 from the Danish Science Foundation and BK Medical Aps, Denmark.

\section{REFERENCES}

[1] J. A. Jensen and N. B. Svendsen. Calculation of pressure fields from arbitrarily shaped, apodized, and excited ultrasound transducers. IEEE Trans. Ultrason., Ferroelec., Freq. Contr., 39:262-267, 1992.

[2] J. A. Jensen. Field: A program for simulating ultrasound systems. In Med. Biol. Eng. Comp., volume 34, pages 351-353, 1996.

[3] J. W. Goodman. Introduction to Fourier Optics. McGraw-Hill, New York, 1968.

[4] P. Alais and P. Y. Hennion. A fourier theory of the nonlinear interaction of acoustical beams in absorbing fluid. the special case of parametric emission. Acustica, 43:1-11, 1979.

[5] Y. Du and J. A. Jensen. Feasibility of non-linear simulation for Field II using an angular spectrum approach. In Proc. IEEE Ultrason. Symp., pages 1314-1317, 2008.

[6] Y. Du, H. Jensen, and J. A. Jensen. Angular spectrum simulation of pulsed ultrasound fields. In 2009 IEEE Ultrason. Symp., pages 2379-2382, 2009.

[7] L. K. Taylor, M. Schlaikjer, and J. A. Jensen. Modeling of higher harmonics formation in medical ultrasound systems. In Med. Imag. V Symp., pages 59-67, 2002.

[8] J. A. Jensen, P. D. Fox, J. Wilhjelm, and L. K. Taylor. Simulation of non-linear ultrasound fields. In Proc. IEEE Ultrason. Symp., volume 2, pages 1733-1736, 2002.

[9] J. Tavakkoli, D. Cathignol, and R. Souchon. Modeling of pulsed finite-amplitude focused sound beams in time domain. J. Acoust. Soc. Am., 104:2061-2072, 1998.

[10] J. P. Remenieras, O. B. Matar, V. Labat, and F. Patat. Time-domain modeling of nonlinear distortion of pulsed finite amplitude sound beams. Ultrasonics, 38:305-311, 2000.

[11] T. Varslot and G. Taraldsen. Computer simulation of forward wave propagation in soft tissue. IEEE Trans. on UFFC, 52(9):1473-1482, 2005.

[12] T. Varslot and S. E. Måsøy. Forward propagation of acoustic pressure pulses in 3D soft biological tissue, modeling, identification and control. IEEE Trans. on UFFC, 27(3):181-200, 2006.

[13] M. F. Hamilton and D. T. Blackstock. Nonlinear Acoustics: Theory and Application. Academic Press, 1998.

[14] P. J. Westervelt. Parametric acoustic array. J. Acoust. Soc. Am., 35(4):535-537, 1963. 
[15] L. E. Kinsler, A. R. Frey, A. B. Coppens, and J. V. Sanders. Fundamentals of Acoustics. John Wiley and Sons, fourth edition, 2000.

[16] B. J. Landsberger and M. F. Hamilton. Second-hamonic generation in sound beams reflected from, and transmitted through, immersed elastic solids. J. Acoust. Soc. Am., 109(2):488-500, 2001.

[17] Y. Xiang and M. F. Hamilton. Angular spectrum decomposition analysis of second harmonic ultrasound propagation and its relation to tissue harmonic imaging. In Ultrasonic and Advanced Methods for Nondestructive Testing and Material Characterization, volume UmassD-NDT 2006, pages 11-24, 2006.

[18] A. D. Polyanin. Handbook of Linear Partial Differential Equations for Engineers and Scientists. Russian Academy of Sciences, 2001.

[19] G. Arfken. Mathematical method for physicists. Academic Press, Orlando, FL, 3rd edition, 1985.

[20] R. Bracewell. The Fourier transform and its applications. McGraw-Hill, New York, 3rd edition, 1999.

[21] Y. Xiang. Statistical Model of Beam Distortion by Tissue Inhomogeneities in Tissue Harmonic Imaging. PhD thesis, The University of Texas at Austin, December 2004.

[22] C. Shannon. Communication in the presence of noise. In Proc. IEEE, volume 37, pages 10-21, 1949.

[23] J. G. Proakis and D. G. Manolakis. Digital signal processing. Pearson Education, Upper Saddle River, New Jersey, 2007.

[24] D. H. Simpson, T. C. Chien, and P. N. Burns. Pulse inversion doppler: a new method for detecting nonlinear echoes from microbubble contrast agents. IEEE Trans. Ultrason., Ferroelec., Freq. Contr., 46(2):372-382, 1999.

[25] M. A. Averkiou. Tissue harmonic imaging. In Proc. IEEE Ultrason. Symp., volume 2, pages 1563-1572, 2000.

[26] J. A. Jensen. Medical ultrasound imaging. Progress in Biophysics and Molecular Biology, 93:153-165, 2007. 